\title{
In reply: Effect of respiratory changes in tracheal length on computed tomographic study of bronchial anatomy
}

\author{
Jean S. Bussières, MD, FRCPC (1) · Yves Lacasse, MD, MSc, FRCPC · Michel Gingras, MD, \\ FRCPC $\cdot$ Étienne J. Couture, MD, MSc, FRCPC $\cdot$ Jacques Somma, MD, \\ BEng, FRCPC
}

Received: 28 August 2019/Revised: 28 August 2019/Accepted: 23 September 2019/Published online: 29 October 2019

(C) Canadian Anesthesiologists' Society 2019

\section{To the Editor,}

We would like to thank Dr. Parida for his comments on our study of the anatomy of the right upper lobe as it relates to the positioning of right-sided double lumen endotracheal tubes. $^{1-4}$ Older studies describing variation in bronchial anatomy with respiratory cycle were cited to highlight concerns with measurement of the tracheobronchial tree. ${ }^{5,6}$ Anatomical measurements of the airway have been obtained in different manners: cine-radiographic bronchography, ${ }^{6}$ cadavers, ${ }^{4}$ resin casts, ${ }^{4}$ and flexible bronchoscopy. ${ }^{4}$ Computerized tomography (CT) with three-dimensional (3-D) reconstruction ${ }^{2}$ has refined measurements in spontaneously breathing patients. Except for the cine-radiographic bronchography and the flexible bronchoscopy, all these measurements are performed in one static period of the respiration cycle.

J. S. Bussières, MD, FRCPC ( $₫)$ · É. J. Couture, MD, MSc, FRCPC · J. Somma, MD, BEng, FRCPC

Department of Anesthesiology and Critical Care, Université Laval, Quebec City, QC, Canada

e-mail: jbuss@criucpq.ulaval.ca

Y. Lacasse, MD, MSc, FRCPC

Multidisciplinary Department of Respiratory Medicine and

Thoracic Surgery, Université Laval, Quebec City, QC, Canada

M. Gingras, MD, FRCPC

Department of Radiology, Institut universitaire de cardiologie et de pneumologie de Québec, Université Laval, Quebec City, QC, Canada
While the addition of dynamic alterations in airway length during the respiratory cycle could be considered, we were obliged to reproduce the methods of Kim et al. to ensure comparability of results. With regard to standard timing of measurement with the respiratory cycle, our protocol specifies that the patients took a deep inspiration and held it for the duration of the CT-scan image acquisition. This protocol is widely used among conscious, spontaneously breathing patients. We appreciate Dr. Parida's concern regarding the training of observers. The two observers in our study regularly use CT-scan reconstruction and the inter-observer agreement documented was substantial with both methods (Kim's method: intraclass correlation coefficient $[$ ICC $]=0.84$; carina-to-carina method: ICC $=0.95$ ), and both measures were closely related (ICC $=0.93 ; P<0.001$ ). Our clinical practice has shown that after a brief training, thoracic anesthesiologists can make these measurements on standard, coronal CT-scans, without 3-D reconstruction. Our experience suggests that clinician measurements approximate the precise measurement within $2 \mathrm{~mm}$. Finally, we mentioned in our article that these measurements apply only to the Broncho-Cath (Mallinckrodt Medical Ltd, Athlone, Ireland) right-sided double lumen tube (R-DLT) as the initial measurements by Kim et al. were done with this model of R-DLT. ${ }^{2}$ The figure shows the different models of R-DLT and it is easy to understand the specificity of Kim's results and consequently of our conclusions.

We sincerely thank Dr. Parida for his comments. We hope our reply will addresses his concerns and allows readers to better apply our findings. 
Figure Cuffs of right-sided double-lumen endobronchial tubes from top to bottom: Mallinckrodt, Portex, Sheridan, and Rusch. Images on left represent tubes as they would be placed in the airway. Images on right provide detail of each tube's bronchial cuff.

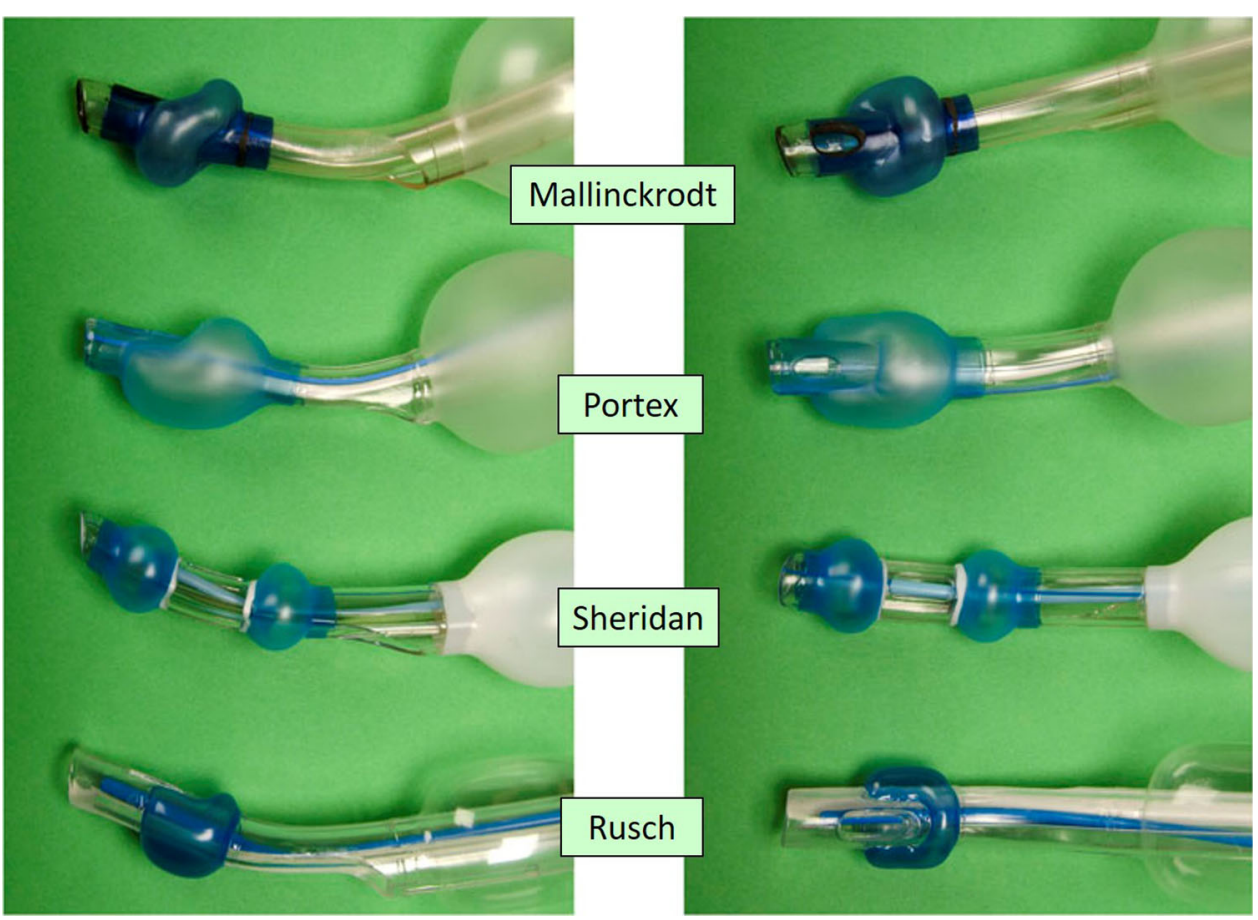

Conflicts of interest None declared.

Editorial responsibility This submission was handled by Dr. Gregory L. Bryson, Deputy Editor-in-Chief, Canadian Journal of Anesthesia.

\section{References}

1. Parida $S$. Effect of respiratory changes in tracheal length on computed tomographic study of bronchial anatomy. Can J Anesth 2020; 67: DOI: https://doi.org/10.1007/s12630-019-01514-5.

2. Kim JH, Park SH, Han SH, Nahm FS, Jung CK, Kim KM. The distance between the carina and the distal margin of the right upper lobe orifice measured by computerised tomography as a guide to right-sided double-lumen endobronchial tube use. Anaesthesia 2013; 68: 700-5.

3. Bussières JS, Gingras M, Perron L, et al. Right upper lobe anatomy revisited: a computed tomography scan study. Can J Anesth 2019; 66: 813-9.

4. Benumof JL, Partridge BL, Salvatierra C, Keating J. Margin of safety in positioning modern double-lumen endotracheal tubes. Anesthesiology 1987; 67: 729-38.

5. Macklin CC. The musculature of the bronchi and lungs. Physiol Rev 1929; 9: 1-60.

6. Holden WS, Ardran GM. Observations on the movements of the trachea and main bronchi in man. J Fac Radiol 1957; 8: 267-75.

Publisher's Note Springer Nature remains neutral with regard to jurisdictional claims in published maps and institutional affiliations. 